\title{
La Belgique: responsable ou bouc émissaire de l'échec des négociations Fouchet?
}

Citation for published version (APA):

Vanhoonacker - Kormoss, S. (1989). La Belgique: responsable ou bouc émissaire de l'échec des négociations Fouchet? Res Publica: politiek-wetenschappelijk tijdschrift van de Lage Landen, 31(4), 513526. https://doi.org/10.21825/rp.v31i4.18859

Document status and date:

Published: 01/01/1989

DOI:

https://doi.org/10.21825/rp.v31i4.18859

Document Version:

Publisher's PDF, also known as Version of record

Document license:

Taverne

Please check the document version of this publication:

- A submitted manuscript is the version of the article upon submission and before peer-review. There can be important differences between the submitted version and the official published version of record.

People interested in the research are advised to contact the author for the final version of the publication, or visit the DOI to the publisher's website.

- The final author version and the galley proof are versions of the publication after peer review.

- The final published version features the final layout of the paper including the volume, issue and page numbers.

Link to publication

\footnotetext{
General rights rights.

- You may freely distribute the URL identifying the publication in the public portal. please follow below link for the End User Agreement:

www.umlib.nl/taverne-license

Take down policy

If you believe that this document breaches copyright please contact us at:

repository@maastrichtuniversity.nl

providing details and we will investigate your claim.
}

Copyright and moral rights for the publications made accessible in the public portal are retained by the authors and/or other copyright owners and it is a condition of accessing publications that users recognise and abide by the legal requirements associated with these

- Users may download and print one copy of any publication from the public portal for the purpose of private study or research.

- You may not further distribute the material or use it for any profit-making activity or commercial gain

If the publication is distributed under the terms of Article $25 \mathrm{fa}$ of the Dutch Copyright Act, indicated by the "Taverne" license above, 


\section{La Belgique : responsable ou bouc émissaire de l'échec des négociations Fouchet?}

par Sophie VANHOONACKER *

Chercheur à l'Institut Européen d'Administration Publique

Le 22 janvier 1988, le président Mitterrand et le chancelier Kohl ont célébré le 25ème anniversaire du traité franco-allemand de l'Elysée. Ce traité de coopération et d'amitié, signé en 1963 par le général de Gaulle et le chancelier Adenauer, est rest en sommeil pendant les premières années de son existence. Mais à partir des années 1970, l'axe franco-allemand se réveille et devient la locomotive du processus ф'intégration européenne. L'exemple le plus significatif à cet égard est l'établissement du Système Monétaire Européen (SME) en 1979 suite à une proposition du chancelier Schmidt et du président Giscard d'Estaing. Des incertitudes croissantes au niveau international font en sorte que la coopération franco-allemande qui, dans le passé, s'est surtout limitée à des initiatives dans le domaine économique, s'aventure dans les années 1980 sur le terrain glissant de la défense. Le poinł culminant en est la signature le 22 janvier 1988 d'un protocole établissant un conseil commun de défense et de sécurité et la constitution d'une brigade franco-łllemande ${ }^{1}$.

Dans cet article, nous remontons aux origines du traité de l'Elysée qui se situent au début des années 1960 lors des négociations sur le projet du général de Gaulle portant sur l'établissement d'une union politique entre les Six. A la suite de la suspension des négociations le 17 avril 1962 causée par le véto de la Belgique et des Pays-Bas et poussée par des événements sur la scène internationale, la France décide de faire cavalier seul avec l'Allemagne. Les fondements du couple franco-allemand sont scellés dans la signature du traité de l'Elysée.

Quant aux raisons de l'échec de la réalisation d'une union politique à Six, les opinions sont encore aujourd'hui partagées. Est-il dû à l'attitude dominatrice de de Gaulle qui considère la coopération politique à Six en premier lieu comme un instrument pour assurer les intérêts et l'influence de la France? Ou faut-il en attribuer la faute à l'attitude intransigeante des Pays-Bas dont le ministre des Affaires étrangèłes, Luns, ne peut pas approuver les ambitions françaises? Ou encore,

\footnotetext{
* Je tiłns à remercier le Professeur Panos Tsakaloyannis pour ses commentaires utiles qui m'ont guidée dáns la rédaction de cet article.

(1) Poür un aperçu historique de la coopération franco-allemande, voir : B. BRIGOULEIX, The Franco-German cement to the E.C. edifice. Dans: European Affairs, 1987, $n^{\circ} 3$, pp. 62-67; W. WEIDENFELD, 25 years after 22 january 1963: The Franco-german Friendship Treaty, Dans: Aussenpolitik, 1988, $\mathrm{n}^{\circ}$ 2, pp. 3-12.

Pour la naissance du S.M.E., voir: P. LUDLOW, The making of the European Monetary System. London, 1982 , p. 63.

Pour la coopération franco-allemande dans le domaine militaire, voir: Military cooperation : Germany-France. Annexe 5. Dans: P. TSAKALOYANNIS, Western european security in a changing world : from the reactivation of the Western European Union to the Single European Act. Maastricht, 1988 , pp. 149-154.
} 
faut-il rechercher la responsabilité chez les Belges et leur ministre des Affaires étrangères, Spaak, qui ont changé de camp au cours des négociations, passant d'une attitude plutôt favorable à un rejet complet du projet. Les études consacrées jusqu'ici au déroulement des négociations se sont surtout concentrées sur le rôle de la France et des Pays-Bas ${ }^{2}$. La position de la Belgique, qui pourtant a joué un rôle important, a moins retenu l'attention. Ceci s'explique probablement par le fait qu'elle est plus complexe et que les motifs qui l'ont dictée ne sont pas toujours clairs. Cet article se propose de combler cette lacune en se livrant à un examen détaillé de l'attitude belge tout au long des négociations ${ }^{3}$.

\section{Le projet français pour une union politique}

Après l'échec du projet de la Communauté européenne de Défense (1954), les Six abandonnent l'approche supranationale et optent, dans les Traités de Rome (1957), pour une réalisation progressive de l'Europe unie par l'intermédiaire des institutions communautaires. Inspirés par la théorie néofonctionnaliste, les auteurs des traités sont convaincus que l'intégration économique mènera automatiquement à l'intégration politique. Cependant, les premières initiatives de coopération politique européenne se situent hors du cadre communautaire et reposent sur la méthode intergouvernementale. En juin 1959, une proposition franco-italienne envisage d'organiser des consultations trimestrielles des ministres des Affaires étrangères des Six sur la politique internationale. Malgré les réticences des Pays-Bas et de la Belgique, la première réunion a lieu le 25 janvier 1960 à Rome.

La proposition lancée par le général de Gaulle lors d'une conférence de presse le 5 septembre 1960 pour établir une 'coopération régulière des Etats de l'Europe

(2) Les trois études principales sur les négociations Fouchet sont :

- R. BLOES, Le 'plan Fouchet' et le probleme de l'Europe politique. Bruges, 1970.

- S. BODENHEIMER, Political Union: A microcosm of European politics, 1960-1966. Leiden, 1977.

- A. SILJ, Europe's political puzzle. A study of the Fouchet negociations and the 1963 veto. Harvard, 1967.

Il faut y ajouter une étude récente sur la position des Pays Bas, faite par H. NIJENHUIS qui, pour la première fois, a pu consulter les archives du ministère des Affaires étrangères à La Haye:

H. NIJENHUIS, De Nederlandse taktiek in de onderhandelingen over een Europese politieke unie (1960-1962): Nee tegen de Gaulle! Dans: Internationale Spectator, 1987, n 1, pp. 41-49.

(3) Pour ce qui concerne les sources consultées, nous nous sommes principalement inspirés des mémoires des principaux acteurs de l'époque (voir ci-dessous), des études déjà publiées au sujet des négociations pour une union politique (voir (2)), des articles de presse ainsi que des communiqués officiels. Etant donné que la loi belge n'autorise la consultation des archives que 30 ans après les fairs, il nous a malheureusement été impossible de consulter les documents du Ministère des Affaires étrangères à Bruxelles. Cependant, les archives du Ministère des Affaires étrangères à La Haye nous ont fourni des informations intéressantes quant aux consultations qui ont eu lieu dans le cadre du Benelux. K. ADENAUER, Erinnerungen 1959-1963. Stuttgart, 1968.

A. CATTANI, Essai de coopération politique entre les Six et échec des négociations pour un statut politique. Dans: Chronique de politique étrangère, $1967, \mathrm{n}^{\circ} 4$, pp. 389-400.

M. COUVE DE MURVILLE, Une politique etrangère, 1958-1969. Paris, 1971.

C. FOUCHET, Mémoires d'hier et de demain. Au service du Général de Gaulle. Paris, 1971.

CH. DE GAULLE, Mémoires d'espoir. S.1., 1970-1971, 2 vol.

H. MACMILLAN, Pointing the way. London, 1972.

J. LUNS, 'Ik herinner mij'. Vrijmoedige herinneringen van Mr. J.M.A.H. Luns zoals verteld aan Michel van der Plas. Leiden, 1971.

P.H. SPAAK, Combats inachevés. Paris, 1969. 
occidentale' continue dans la même voie de l'approche intergouvernementale 4. Cette initiative par laquelle de Gaulle se pose en promoteur de la coopération politique européenne, doit être mise en rapport avec le mémorandum français du 17 septembre 1958. Dans ce mémorandum, de Gaulle, estimant que l'organisation de l'alliance occidentale ne correspond plus aux conditions nécessaires de la sécurité' 5 , adresse au président américain Eisenhower et au Premier ministre anglais Macmillan la proposition d'établir 'un directoire à trois dans le domaine de la politique internationale et de la stratégie'. Mais les deux autres partenaires ne sont pas enthousiasmés par les ambitions gaulliennes qui visent à acquérir une place privilégiée, d'égal à égal avec la Grande-Bretagne et les Etats-Unis. Eisenhower, qui estime inconcevable de partager à trois la position dominante des Etats-Unis, se tire d'affaire en répondant qu'il ne serait pas possible 'd'adopter un système qui donnerait aux autres alliés ou à d'autres pays du monde libre l'impression que des décisions fondamentales affectant leurs propres intérêts vitaux sont prises sans leur participation' ${ }^{6}$. Il ne reste à la France qu'à se tourner vers l'Europe pour essayer d'acquérir ce statut de puissance mondiale qu'elle ambitionne tant. Mais il n'est pas évident que les cinq autres partenaires européens seront prêts à lui accorder le rôle qu'elle revendique. Au cours des négociations, il devient clair que plusieurs d'entres eux, plus particulièrement les petits pays, bien que favorables à la coopération politique, ne sont pas prêts à laisser la France devenir le porte-parole de l'Europe.

Conłme instrument principal pour organiser la coopération à Six, de Gaulle propose des réunions régulières où les chefs d'Etat et de gouvernement se concerterapent pour harmoniser leurs actions dans les domaines politique, économique, culturel et de la défense. Selon ce projet, des organismes specialisés, aidés par un secrétariat permanent à Paris, feraient le travail préparatoire. Une assemblée européenne composée de délégués de parlements nationaux serait habilitée à faire des recommandations. Et un référendum donnerait à la coopération l'appui indispensable du peuple européen. Avant de lancer son projet, de Gaulle a procédé à des consultations bilatérales avec les gouvernements de l'Allemagne, des PaysBas et de l'Italie, qui font tous preuve d'une grande réserve. Le fait que la Belgique et le Laxembourg ne seront consultés qu'après sa conférence de presse est révélateur du style de de Gaulle. Tout au long des négociations, les petits pays sentiront très nettement que leur avis ne vient qu'en deuxième position.

Lors de leur visite à l'Elysée le 13 septembre 1960, le Premier ministre belge, Eyskens, accompagné de son ministre des Affaires étrangères, Wigny ${ }^{7}$, prêtent une ortille complaisante à l'idée d'une coopération politique européenne tout en soulignant qu'ils ne soutiendront le projet que si la nouvelle organisation ne porte pas atteinte à l'OTAN et aux compétences communautaires. Le lendemain, Wigny

(4) CH. DE GAULLE, Discours et messages. Avec le renouvean. Mai 1958-juillet 1962. Paris, 1970, pp. 244-246. 202.

(5) Pour le texte de la lettre de de Gaulle, voir : A. GROSSER, Les Occidentaux. Paris, 1978, p.

(6) Pour la réponse de Eisenhower, voir : L. PATTISON DE MENIL, Who speaks for Europe? The vision of Charles de Gaulle. London, 1977, pp. 193-194.

(7) Au début des négociations pour une union politique la Belgique était gouvernée par la coalition de chrétiens démocrates-libéraux Eyskens-Lilar (6/11/1958-27/3/1961). 
demande des informations supplémentaires sur les intentions françaises dans un entretien avec son collègue Couve de Murville. L'inquiétude de Wigny sur l'affaiblissement de l'OTAN et la CEE est quelque peu apaisée. En ce qui concerne la participation de la Grande-Bretagne à l'organisation projetée, la France estime que l'adhésion préalable aux Communautés est indispensable. Wigny s'abstient de toute prise de position mais promet d'étudier la proposition dans un esprit ouvert ${ }^{8}$.

\section{Le sommet de Paris et les travaux de la commission préparatoire}

La proposition française est discutée pour la première fois au niveau des Six lors du sommet des chefs d'Etat et de gouvernement à Paris, les 10 et 11 février 1961. L'active diplomatie française a réussi à atténuer les réticences exprimées antérieurement et, lors de la réunion, tous les Etats membres, à l'exception des Pays-Bas, se montrent favorables à l'initiative. Le ministre hollandais des Affaires étrangères, Luns, s'oppose radicalement à l'établissement d'une union politique européenne dont la Grande-Bretagne serait exclue. La tentative de son homologue Wigny qui, en faisant référence au Benelux, essaie de lui faire adopter une position plus flexible, reste sans résultat. On comprend mieux l'attitude conciliante adoptée par la Belgique lors du sommet de Paris si l'on tient compte des problemes que le pays connaît à cette époque au Congo. Après la proclamation de l'indépendance du Congo le 30 juin 1960, l'ancienne colonie belge est confrontée à des mutineries dans la force publique et à la sécession de la riche province minière du Katanga. L'intervention militaire et civile de la Belgique qui a encore des intérêts considérables dans le pays est fortement critiquée au niveau international. Au sein des Nations unies, la France est le seul pays à soutenir la politique belge. Sa situation précaire et sa dépendance de l'aide française mettent la Belgique dans une position où elle peut difficilement se permettre d'adopter une attitude trop critique envers les propositions de de Gaulle pour une union politique ${ }^{9}$. Un deuxième élément qui a probablement atténué les réserves est de caractère plus personnel. Couve de Murville aurait notamment suggéré à son homologue Wigny de prendre la direction du secrétariat permanent qui serait établi à Paris. La perspective des élections anticipées qui se dessinent dans le paysage politique belge n'aurait pas été étrangère à l'attitude bienveillante de Wigny ${ }^{10}$.

Le texte du communiqué adopté à Paris est clairement le résultat d'un compromis ${ }^{11}$. Il fait référence aux traités de Rome et de Paris, et exprime le désir

(8) Archives du Ministère des Affaires étrangères, La Haye, nº 912.2.

(9) Pour la Belgique et l'indépendance du Congo, voir:

R. COOLSAET, Buitenlandse Zaken. Leuven, 1987, pp. 170-174.

Th. LUYKX, Politieke geschiedenis van België. Amsterdam-Brussel, 1978, pp. 498-501.

La politique 'Katangaise' de la Belgique. Dans: Courrier hebdomadaire, 1963, $\mathrm{n}^{\circ} 182$.

Les problemes du Congo devant le Conseil de sécurité des Nations unies. Dans: Courrier hebdomadaire, $1960, \mathrm{n}^{\circ} 179$.

(10) Archives du Ministère des Affaires étrangères, La Haye, $\mathrm{n}^{\circ}$ 913.10.

H. NIJENHUIS, o.c., pp. 44-45.

(11) Le dossier de l'Union politique. Recueil de documents avec préface de E. Battista. Paris, 1964, pp. 6-7.

Pour tous ceux qui étudient les négociations Fouchet ce dossier, qui contient tous les principaux textes à ce sujet, constitue un ouvrage de référence indispensable. 
de développer les échanges avec la Grande-Bretagne et de coopérer avec les EtatsUnis. Suite à la proposition des Pays-Bas, une commission composée de représentants des six gouvernements est chargée d'élaborer des propositions de coopératiфn politique européenne. Sur l'insistance de la Belgique, cette commission se voit également confier la tâche d'étudier 'les problèmes en rapport avec les développements des Communautés'.

Lors d'une allocution devant l'Assemblée parlementaire à Strasbourg le 9 mars 1961 ,Wigny se montre très positif au sujet du sommet de Paris et il met beaucoup d'espoir dans les travaux de la commission préparatoire ${ }^{12}$. La première réunion de cette commission a lieu à Paris les 16 et 17 mars et est présidée par le Français Fouchet, ancien ambassadeur au Danemark. La Belgique est représentée par le baron Holvoet, directeur général de la Politique. La position des Pays-Bas reste inchar|gée. Ils continuent à refuser tout engagement quant à l'institutionnalisation de la doopération politique sans garanties préalables pour les compétences communautaires et la participation de la Grande-Bretagne aux réunions des chefs d'Etat et de gouvernement. Le seul cadre dans lequel les problèmes internationaux peuvent être traités est celui de l'Alliance atlantique. Pendant une réunion des ministres des Affaires étrangères le 18 mars 1961 à Bruxelles, les pays Benelux essaient de rapprocher leurs points de vue mais leurs efforts sont vains ${ }^{13}$. Au sein de la §ommission préparatoire les travaux avancent beaucoup moins vite qu'on l'aurait voulu et on est obligé de reporter d'un mois le deuxième sommet prévu pour le 19 mai 1961.

Il faut signaler qu'à cette époque, un nouveau gouvernement composé de Démocrates-Chrétiens et Socialistes accède au pouvoir en Belgique. Dans un premier temps, ce changement n'a pas de répercussions sur la politique belge au sein de la commission Fouchet. Le Premier ministre chrétien-démocrate Lefèvre, qui avait qualifié les propositions de de Gaulle comme insuffisantes pour parvenir à une politique européenne en septembre $1960^{14}$, se montre beaucoup plus favorable dans la déclaration gouvernementale du 2 mai 1961. Il y affirme notamment qu'il est 'disposé à collaborer à toute relance européenne dans le domaine politique' 15 . De mêtme, le vice Premier ministre et ministre des Affaires étrangères, le socialiste Spaak, s'exprime favorablement sur une relance politique européenne. Spaak a été absent de la scène politique belge pendant quatre ans pour occuper le poste de Secrétaire général à l'OTAN (16 mai 1957 - 5 mars 1961). Plus que quiconque il est conscient de la nécessité d'une véritable consultation politique, aussi bien au niveau européen qu'au niveau atlantique. A l'OTAN, le développement d'un échange de vues régulier entre alliés a été un de ses principaux champs d'action ${ }^{16}$. Lors de son discours devant la Chambre belge des représentants, le 14 juin 1960 , il affirme qu'il est 'en principe assez d'accord sur les propositions faites par le gé-

(12) L'Année politique en Europe 1961. Paris, 1962, p. 368

(13) Archives du Ministère des Affaires étrangères, La Haye, $n^{\circ} 913.100$.

(14) Conférence aux 'Nouvelles Equipes Internationales'. Dans: Agence Europe, 22 septembre 1960.

(15) La politique extérieure belge, mai 1961-mai 1962. Dans : Courrier Hebdomadaire, 1962, $\mathrm{n}^{\circ}$ 150 , p. 15 .

(16) La pensée européenne et atlantique de Paul-Henri Spaak, 1942-1972. Textes réunis et présentés par P.F. Smets. Bruxelles, 1980, vol. 2, p. 791. 
néral de Gaulle' ${ }^{17}$. Il est clair que pour le supranationaliste Spaak qui a présidé la rédaction de l'avant-projet du traité réalisant une Communauté Politique Européenne (octobre 1952 - mars 1953), le projet français est loin d'être idéal. Si Spaak l'accepte, ce n'est que parce qu'il n'y a pas d'autres initiatives à ce moment. Il est préparé à collaborer à condition que les compétences communautaires soient intégralement respectées et qu'aucune décision ne soit prise en matière de politique militaire sans consulter la Grande-Bretagne et les Etats-Unis ${ }^{18}$. Malgré ces réticences, Spaak se montre très optimiste quant au travail fait par la commission préparatoire : ‘... je pense que nous allons arriver à un accord dans les semaines qui viennent et que nous serons alors entrés dans une voie nouvelle de la construction européenne ${ }^{19}$. Lefèvre et Spaak qui accompagnent le roi et la reine des Belges lors de leur visite officielle à Paris le 25 mai 1961, réaffirment leur soutien au cours d'un entretien avec leurs homologues Debré et Couve de Murville. Dans une déclaration faite à l'issue de cet entretien, Spaak constate que les points de vue des deux partenaires 'sont communs sur de nombreux points' 20.

Au niveau du Benelux, au contraire, il semble moins facile d'harmoniser les positions. A la conférence des ministres des Affaires étrangères des trois pays, le 5 juin 1961, les Pays-Bas font une deuxième tentative ${ }^{21}$ pour sensibiliser leurs partenaires à leur attitude réticente envers le projet français et au préalable anglais. A titre de compris la Belgique et le Luxembourg acceptent d'inviter les Anglais aux consultations politiques et les Pays-Bas acceptent que l'on y discute de questions concernant l'OTAN 22. Spaak propose de consigner les résultats de la réunion dans un mémorandum. Mais il ne respecte pas tout à fait les concessions qu'il a faites le 5 juin : la participation anglaise y est seulement présentée comme souhaitable; les problèmes qui ressortissent à l'OTAN ne peuvent pas seulement être discutés mais peuvent être également décidés après consultation préalable avec les autres membres de l'Alliance. Les discussions peuvent même porter sur l'économie communautaire à condition que les Communautés soient représentées ${ }^{23}$. Plusieurs consultations ont encore lieu entre les Pays-Bas et la Belgique mais finalement le mémorandum belge est accepté comme base de la position du Benelux. Par conséquent, à la conférence des ministres des Affaires étrangères à Rome le 10 juillet, les pays Benelux peuvent enfin parler d'une seule voix.

\section{Le sommet de Bonn}

Au deuxième sommet qui a lieu à Bonn le 18 juillet 1961 , le principe des réunions périodiques au niveau des chefs d'Etat et de gouvernement est finalement accepté par tous les six Etats membres. La commission préparatoire reçoit le mandat de faire des propositions qui permettent de donner un caractère statutaire à

(17) Ibidem, p. 805 .

(18) Ibidem, pp. 807-808.

(19) Ibidem, p. 808.

(20) La politique extérieure belge, mai 1961-mai 1962. Dans: Courrier Hebdomadaire, 1962, $\mathrm{n}^{\circ}$ 150 , p. 6 .

(21) Cfr. supra, p. 5.

(22) Archives du Ministère des Affaires étrangères, La Haye, $\mathrm{n}^{\circ}$ 913.100.

(23) Archives du Ministère des Affaires étrangères, La Haye, $n^{\circ} 913.100$. 
l'union des peuples. A titre de compromis, le communiqué fait également référence au renforcement de l'Alliance atlantique et aux Traités de Rome ${ }^{24}$. Contrairement aux réactions positives et optimistes avec lesquelles le communiqué de Bonn est accueilli par la presse et l'opinion publique, les deux ministres belges représentés au sommet sont beaucoup plus sceptiques. Dans un interview accordé au journal 'Le Monde' quelques jours plus tard, Lefèvre déclare que 'la réunion de Bonn n'avait pas eu tous les résultats désirables parce que l'un des partenaires n'a décidáment pas l'esprit européen' 25 . Bien qu'il ne cite pas ledit partenaire nommément, sa référence à la France est évidente. Le ministre Spaak également, s'inquiète de l'attitude française. A Bonn, il se rend compte que les deux conditions ${ }^{26}$ dont il avait assorti son appui au projet sont loin d'être remplies. Pour ce qui est des Communautés, il comprend que la France a l'intention de réduire son action 'au strict minimum, un minimum purement technique et de lui enlever toute action politique'. L'insistance française sur la particularité des problèmes de défense des Six et le désir de de Gaulle d'arriver à une sorte "d'entente militaire européenne", résonnent comme une hérésie aux oreilles de l'atlantiste Spaak. Les Néerlandais sont les seuls à partager son inquiétude et à l'appuyer dans ses observations. Pour Spaak les textes publiés à l'issue du sommet ne sont pas du tout représentatifs de l'aumosphère qui y a régné ${ }^{27}$.

\section{Le plan Fouchet I}

A la première réunion de la Commission Fouchet après le sommet de Bonn, les délegués allemands, belges, italiens et luxembourgeois présentent chacun un document avec des propositions. Les Pays-Bas pour qui contrarier de Gaulle est plus important que jouer un rôle constructif, sont les seuls à arriver les mains vides. La Belgique propose l'institution progressive d'une Communauté politique au cours d'une période de dix ans. Les premières cinq années seraient consacrées à la coordination des politiques étrangères et de l'action dans le domaine culturel et de la recherche scientifique. La deuxième étape verrait l'élaboration d'une véritable politique extérieure commune ainsi que d'une politique commune dans tous les domaines étrangers à celui des CEE. Les institutions prévues sont un conseil des ministres, assisté d'une commission de suppléants (pouvoir exécutif), une assemblée parlementaire et une cour de justice. On envisage aussi d'intégrer les Communautés existantes dans la nouvelle structure ${ }^{28}$. Mais les propositions belges sont trop ambitieuses pour servir de base de négociation et c'est le projet plus modéné présenté par Fouchet le 19 octobre 1961 qui est retenu. L'union d'Etats proposée dans ce projet a des compétences dans les domaines de la politique étrangère, de la politique des sciences et de la culture, et de la politique de la défense. Au niveau institutionnel, il y a un conseil composé des chefs d'Etat et de gouvernement, une commission politique composée de hauts fonctionnaires et une

(24) Le dossier de l'Union politique. Recueil de documents avec préface de E. Battista. Paris, 1964, pp. $9-10$.

(25) Le Monde, 21 juillet 1960.

(26) Cfr. supra, p. 6.

(27) La pensée européenne et atlantique de Paul-Henri Spaak, 1942-1972. Textes réunis et présentés plar P.F. Smets. Bruxelles, 1980 , vol. 2, p. 811.

(28) Archives du Ministère des Affaires étrangères, La Haye, n 913.10. 
assemblée parlementaire. Le traité est ouvert à chaque Etat préalablement membre des Communautés européennes. Il peut être révisé après trois ans afin de renforcer l'union ${ }^{29}$. La première discussion sur ce projet, mieux connu sous le nom de 'Fouchet I', a lieu le 10 novembre 1961 .

Entretemps, il s'est ajouté aux négociations un élément qui sera déterminant pour leur déroulement. Les négociations sur l'adhésion britannique aux CEE commencent le 8 novembre 1961, après que la demande ait été introduite par le gouvernement conservateur de Macmillan le 31 juillet 1961. La revendication des Pays-Bas d'inviter la Grande-Bretagne aux négociations pour une union politique perd enfin son caractère illusoire. Pour la Belgique qui jusqu'alors a refusé d'imposer la participation anglaise comme une condition sine qua non, le préalable anglais devient maintenant une revendication réaliste. A la réunion de la Commission Fouchet du 10 novembre, la Belgique soutient pour la première fois les Pays-Bas. Les quatre autres délégations ne sont pas prêtes à les suivre dans leur demande. La Grande-Bretagne non plus ne partage pas l'enthousiasme des deux pays. Lors de son entretien avec Spaak le 30 novembre 1961, Lord Home fait savoir que les Anglais se contentent d'être tenus au courant de l'évolution des negociations. Ceci explique que la Belgique adopte une position plus flexible lors de la réunion des ministres des Affaires étrangères à Paris le 15 décembre 1961 . Après que les cinq pays lui aient donné l'assurance que l'adhésion aux Communautés économiques impliquera automatiquement la participation à la coopértion politique, Spaak accepte le principe selon lequel les Anglais seront simplement informés de l'état d'avancement du travail de la commission préparatoire ${ }^{30}$. Cependant, un discours de Spaak devant l'Assemblée des Syndicats libres à Bruxelles, le 10 janvier 1962, remettra en cause son attitude conciliante. L'Europe des patries y est qualifiée comme 'une notion étriquée et insuffisante' ${ }^{31}$.

\section{Le plan Fouchet II}

A la réunion de la commission préparatoire du 18 janvier 1962, Fouchet, à la grande surprise de tous, présente une deuxième version du projet qui, sur plusieurs points, constitue un pas en arrière par rapport au compromis atteint au mois de décembre : l'union reçoit des compétences dans le domaine économique et aucune référence n'est faite à l'Alliance atlantique ni à l'adhésion de nouveaux membres ${ }^{32}$. Chez toutes les cinq délégations le brutal revirement français cause l'effet d'un choc et leur réaction au nouveau projet est négative. Pour la première fois ils unissent leurs forces et ils rédigent une contreproposition. On ne sait pas très bien quelles ont été les intentions derrière le nouveau projet français ni le rôle joué par de Gaulle. Alexander Silj parle de 'la phase la plus mystérieuse des négociations Fouchet' ${ }^{33}$. En tout cas l'événement évoque de sérieuses questions sur

(29) Le dossier de l'Union politique. Recueil de documents avec préface de E. Battista. Paris, 1964, pp. $11-14$.

(30) L'Année politique 1961. Paris, 1962, p. 622.

(31) Agence Europe, 10 janvier 1963.

(32) Le dossier de l'Union politique. Recueil de documents avec préface de E. Battista. Paris, 1964, pp. $17-23$.

(33) A. SILJ, o.c., p. 13. 
la volonté de la France de vraiment voir aboutir les négociations. Des pays comme la Belgique qui ont toujours essayé de jouer un rôle conciliateur se retrouvent dans une position difficile et doivent certainement se poser des questions sur l'utilité de la dontinuation de leurs efforts. Pour les Pays-Bas, au contraire, Fouchet II constitue un bon argument supplémentaire pour s'opposer à la réalisation du projet.

Lorts du conseil des ministres qui se tient à Luxembourg le 20 mars 1962, trois points de divergence dominèrent la discussion : les rapports de l'union avec l'Alliance latlantique, ses compétences dans le domaine économique et les conditions de révision du Traité. Dans ses mémoires, Spaak écrit qu'à la fin de la réunion, les ministres se séparent 'découragés ayant décidés cependant de se retrouver à Paris un mois plus tard' 34.

Un semaine avant cette nouvelle réunion, un changement inattendu dans l'attitude anglaise bouleverse le cours des événements. Devant le conseil de l'UEO, Edwatd Heath, le ministre anglais responsable des négociations avec les Six fait savoir qu'il serait souhaitable que la Grande-Bretagne participe aux discussions sur la structure politique future de l'Europe ${ }^{35}$. Le moment que les Pays-Bas ont attench depuis si longtemps est enfin arrivé : ils sont finalement soutenus dans le préłlable anglais par les Anglais eux-mêmes. Pour la Belgique il n'y a maintenant plus aucune raison d'exclure la Grande-Bretagne. Les positions se sont clairement endurcies : la France par le lancement du plan Fouchet II, la Grande-Bretagne et la Belgique par leur appui au préalable anglais.

A la conférence des ministres des Affaires étrangères à Paris le 17 avril 1962, Spaak soutenu par Luns, fait savoir que la Belgique ne signera pas le traité établissant l'union politique tant que la Grande-Bretagne n'aura pas adhéré aux Communattés. Sur les trois problèmes discutés au Luxembourg, le désaccord persiste. La réunion se termine sans fixer un nouveau rendez-vous, sans communiqué et sans donner un autre mandat à la commission préparatoire. La suspension des négociations n'implique pas pour autant que les efforts pour réaliser une union politique à Six sont abandonnés. Dans une lettre ouverte au journal 'Le Soir' 36 Spaak refuse d'accepter l'échec des négociations et il espère qu'un accord sera encore possible. Il s'oppose avec véhémence aux réactions négatives dans la presse selon lesquelles la Belgique et les Pays-Bas sont accusés d'être les principaux responsables. Il explique qu'il n'adopte pas une attitude contradictoire en défendant en mêtme temps la conception de l'Europe supranationale et l'adhésion britannique. Il ne renonce pas du tout à l'idéal d'une Europe supranationale. Mais, conscient de la difficulté de la réaliser à court terme, il est prêt à accepter dans un premicr temps la formule d'une Europe des patries à condition que ce soit une Europe à sept. Seul le poids de la Grande-Bretagne peut contrebalancer l'influence française et allemande 37.

Le 24 juillet 1962, après avoir contacté ses homologues hollandais et italiens et le chancelier Adenauer, Spaak essaie de relancer les négociations en écrivant

(34) P.H. SPAAK, o.c., p. 368.

(35) Le dossier de l'Union politique. Recueil de documents avec préface de E. Battista. Paris, 1964, p. 37.

(36) Le Soir, 20 avril 1962.

(37) P.H. SPAAK, o.c., pp. 368-371. 
au Général de Gaulle qu'il est prêt à laisser tomber le préalable anglais. Il propose d'organiser la coopération politique à l'exemple du Marché commun, c'est-à-dire en instituant une commission politique indépendante, garante des intérêts communautaires. Mais l'idée d'un dialogue entre les institutions communautaires et les gouvernements ne peut emporter l'accord du général. Spaak écrit dans ses mémoires: 'Le ton plus encore que le fond de la réponse du Général de Gaulle me fit comprendre l'inutilité de poursuivre mon effort' 38 .

Finalement, le projet reçoit son coup de grâce définitif le 14 janvier 1963 lors du véto du général de Gaulle à l'entrée de la Grande-Bretagne dans la CEE et de la signature du traité franco-allemand une semaine plus tard, les deux éléments n'étant pas sans rapport.

\section{Le revirement belge}

Après cet aperçu historique et chronologique des négociations Fouchet, il importe de prendre du recul et d'examiner de plus près la position belge et son évolution. Pour mieux comprendre les motifs qui sont à sa base, il est nécessaire de la situer dans le cadre général de la politique étrangère belge.

Depuis la deuxième guerre mondiale cette politique, caractérisée par une grande continuité, repose sur les deux piliers de l'Alliance atlantique et de l'intégration européenne. Après l'échec d'une politique de neutralité, l'adhésion à l'Alliance atlantique devient une des bases essentielles de la sécurité belge. Sa situation géographique et la dépendance de son économie du commerce extérieur font que la Belgique est dès le début un des grands partisans de l'intégration européenne ${ }^{39}$.

Lorsque le général de Gaulle lance l'idée d'une Europe politique, la Belgique, qui considère les Communautés économiques comme une simple étape vers l'Europe unie, ne peut que réagir favorablement à l'initiative. Cependant, les ministres belges qui connaissent très bien l'attitude du général envers l'Alliance atlantique et les CEE, expliquent que le projet ne peut emporter leur approbation que si les compétences atlantiques et communautaires sont intégralement respectées. L'accès au pouvoir du nouveau gouvernement Lefèvre-Spaak n'affecte pas la position belge. Contre toute attente, les Pays-Bas trouvent même en Spaak un défenseur plus fervent du projet que ne l'a été son prédécesseur ${ }^{40}$.

Bien que les Néerlandais aient à différentes reprises essayé de rallier les Belges à leur point de vue, le changement dans l'attitude belge a moins à voir avec leur force de persuasion qu'avec l'apparition d'événements importants sur la scène européenne et internationale. Un premier élément important est la demande d'ad-

(38) P.H. SPAAK, o.c., p. 368.

(39) Pour les bases de la politique étrangère belge, voir :

La Belgique et la Communauté Européenne. Textes et documents. Collection Idées et Etudes. Bruxelles, 1979.

R. COOLSAET, Buitenlandse Zaken. Leuven, 1987.

C. FRANCK, La politique de sécurité de la Belgique. Dans: Studia diplomatica, 1984, n²1, pp. 3139 .

H. SIMONET, Une politique étrangère de la Belgique : hasard ou nécessité. Dans : Studia diplomatica, $1984, \mathrm{n}^{\circ} 1$, pp. $463-482$.

(40) Archives du Ministère des Affaires étrangères, La Haye, $n^{\circ}$ 912.1. 
hésion britannique et l'ouverture des négociations en novembre 1961. Suite à ces développements, la Belgique, grand partisan de cette adhésion, décide de soutenir les Pays-Bas dans leur revendication portant sur la participation britannique aux négociations pour une union politique. En tant que petit pays la Belgique craint la domination de l'union par le couple franco-allemand et elle estime par conséquent que la Grande-Bretagne constituerait un bon contrepoids. En outre, les contacts privilégiés de la Grande-Bretagne avec les Etats-Unis seraient une garantie pour le respect des intérêts atlantiques au sein de l'union. Puisque ni la France, ni la Belgique et les Pays-Bas ne sont prêts à faire des concessions sur la revendication du préalable anglais, cette dernière sera finalement à l'origine de la suspension des négociations.

Il serait incomplet de présenter le problème de l'adhésion britannique comme le seul point de divergence entre la Belgique et la France. A partir de novembre 1961, la Belgique ne revendique pas seulement la participation anglaise aux négociations, mais elle adopte également une attitude plus critique quant à la proposition même. Dans un discours devant le National Press Club, Washington, le 21 novembre 1961, Spaak reproche au projet d'être insuffisant ${ }^{41}$.

Ce changement dans l'attitude de Spaak peut seulement être compris si l'on se réfère aux divergences de vue qu'il y a à cette époque entre Spaak et de Gaulle. Bien que Spaak soit dès le début très conscient que ses idées sur l'Alliance atlantique et l'intégration européenne sont nettement différentes de celles de de Gaulle, il croit que l'Union politique à Six permettrait d'assouplir quelque peu l'attitude de la France et de remettre ce pays sur les rails de l'OTAN ${ }^{42}$. La position française sur lą question de Berlin lui fait vite perdre cette illusion. Lorsque aux réunions du Conseil atlantique en septembre et décembre 1961, Spaak défend les propositions du Kremlin sur Berlin comme une base valable de discussion, il se voit opposer un véto insurmontable de la part de la France ${ }^{43}$. Puisque chaque décision du Conseil atlantique requiert l'unanimité, le refus d'un seul pays suffit pour bloquer toute action. Spaak se rend compte que l'on rencontrera exactement le même problème au sein de la coopération politique telle que présentée dans le plan Fouchet. Aussi longtemps que la règle de la majorité ne prévaut pas, tout fonctionnement efficace est exclu ${ }^{44}$ et les petits pays devront toujours s'incliner devant les voeux des grands. Seule une Europe organisée supranationalement peut leur donner les garanties nécessaires ${ }^{45}$. Tout au long des négociations, Spaak a pu constater que de Gaulle ne prend pas les petits états au sérieux ${ }^{46}$. En supposant qu'il suffit d'obtenir l'accord de l'Allemagne pour assurer le succès de ses propositions, de Gaulle a clairement sousestimé le rôle des petits et les faits du 17 avril

(41) L'année politique 1961. Paris, 1962, p. 597.

(42) Archives du Ministère des Affaires étrangères, La Haye, $n^{\circ} 913.100$.

(43) P.H. SPAAK, o.c., pp. 334-342.

(44) P.H. Spaak dans un discours devant le Chambre belgo-américaine, 25-1-1962. Dans : La pensée eutopéenne et atlantique de Paul-Henri Spaak, 1942-1972. Textes réunis et présentés par P.F. Smets. Bruxelles, 1980, vol. 2, p. 826.

(45) P.H. Spaak dans un entretien avec Paul Winkler et Charles Ronsac pour Opera Mundi, mai 1962. Dans: La pensée européenne et atlantique de Paul-Henri Spaak, 1942-1972. Textes réunis et présentés par P.F. Smets. Bruxelles, 1980, vol. 2, p. 826.

(46) Cfr. supra, p. 3. 
l'ont démontré. Il est significatif que, lorsque en 1981, le président français Mitterrand prend l'initiative de relancer l'UEO, il commence ses délibérations à La Haye tout en veillant à tenir au courant Bruxelles ${ }^{47}$.

Bien que la suspension des négociations le 17 avril soit due aussi bien au véto de la Belgique qu'à celui des Pays-Bas, on ne peut pas du tout mettre les deux pays sur le même niveau. Les Pays-Bas se sont opposés aux propositions françaises dès le début et ont participé aux négociations dans le seul but de ralentir et de bloquer le projet autant que possible, tandis que le rôle de la Belgique a été plus constructif. Sa position parfois contradictoire est due au fait qu'elle a essayé de concilier deux positions incompatibles. D'un côté, elle veut promouvoir l'intégration européenne et appuyer la coopération politique. D'un autre côté, elle ne peut à aucun prix accepter la mise en cause de la solidarité atlantique qui est la base même de sa sécurité. Le plan Fouchet II qui met en cause la volonté de la France de voir aboutir les négociations et les grandes tensions qui règnent à ce moment sur la scène internationale feront finalement pencher la balance du côté atlantique. Pour la Belgique, l'ajournement sine die des négociations n'est pas ressenti comme une victoire, loin s'en faut. Les tentatives de Spaak pour relancer les discussions l'illustrent bien. Mais ces efforts restent sans résultat.

Nous voici arrivés au coeur de la question: Spaak était-il vraiment en mesure de sauver le projet? Il nous paraît que, vu les tensions internationales et les frictions au sein de l'Alliance, les chances de reprendre les discussions étaient très limitées. Entre avril 1962 et janvier 1963, la scène internationale connaît plusieurs événements qui sont déterminants pour l'issue définitive des négociations et sur lesquels Spaak n'a aucune prise. Le thème qui domine l'actualité de l'époque est le débat sur la sécurité de l'Europe ${ }^{48}$. Depuis que, en 1957, l'Union Soviétique a réussi à lancer Spoutnik I, les Etats-Unis se retrouvent pour la première foiş dans leur histoire exposés au risque d'une attaque stratégique et la doctrine des représailles massives est remise en question. A la réunion du conseil atlantique à Athènes en mai 1962, le secrétaire de la Défense américain Mcnamara présente comme alternative la stratégie de la réplique souple. Selon cette stratégie, les EtatsUnis disposeraient de plusieurs options pour répondre à une éventuelle attaque. Les Européens comprennent qu'en d'autres termes, ils ne pourront plus compter sur la garantie absolue nucléaire américaine. Pour apaiser les réactions d'inquiétude en Europe et pour ne pas perdre le contrôle sur la prolifération des armes nucléaires, les Etats-Unis insistent sur l'idée du développement d'une force nucléaire multilatérale (MLF) au sein de l'OTAN. Mais le projet ne plaît pas aux partenaires tels que la France et la Grande-Bretagne qui ont déjà décidé de développer leur propre 'force de frappe'. La Grande-Bretagne craint que le MLF ne constitue une menace pour ses relations privilégiées avec Washington. La France,

(47) J.M. HOSCHEIT, P. TSAKALOYANNIS, The relaunching of the WEU : an overview. Dans : $P$. TSAKALOYANNIS, The reactivation of the WEU : the effects on the EC and its institutions. Maastricht, 1985, p. 15 .

(48) Pour les tensions internationales et les frictions au sein de l'Alliance, voir :

L. FREEDMAN, The evolution of nuclear strategy. London, 1981.

R.E. NEUSTADT, Alliance politics. New York-London, 1970.

L. PATTISSON DE MENIL, Who speakes for Europe? The vision of Charles de Gaulle. London, 1977.

D.N. SCHWARTZ, Nato's nuclear dilemma's. Washington, 1983. 
qui depuis la crise de Suez (1956) a décidé de ne plus dépendre des garanties nucléaines américaines, estime que la possession d'un armement nucléaire propre est indispensable pour avoir un poids au niveau mondial. Elle ressent la nouvelle stratégie américaine comme un réel défi pour sa force de frappe et pour cette raison elle la considère inacceptable. La crise cubaine du 22 octobre 1962, qui pour la première fois dans l'histoire de l'après-guerre montre que la menace d'une guerre nucléaire entre les deux superpuissances est réelle, renforce encore la France dans ses convictions. Pendant que la France essaie d'assurer sa sécurité en optant radicalement pour une indépendance aussi grande que possible, la Grande-Bretagne resserre davantage ses liens avec les Etats-Unis. Lors d'une rencontre entre Kennedy et Macmillan le 18 décembre 1962 à Nassau aux îles Bahamas, les Britanniques obtiennent l'accord américain pour la fourniture de missiles Polaris. Pour de Gqulle, le fait que Kennedy ait cédé à Macmillan indique clairement que la prop $\phi s i t i o n$ d'un MLF n'est qu'un subterfuge supplémentaire pour réduire la France à un statut d'impuissance. Cela vient renforcer la conception gaulienne qui voit la Grande-Bretagne comme un cheval de Troie. Pour lui, l'adhésion britannique aux Communautés ne servirait que comme moyen pour mieux adapter le processus d'intégration européenne aux intérêts américains et pour appuyer la réalisation du 'Grand Design' selon lequel Kennedy veut établir 'une association atlantique' entre l'Europe et les Etats-Unis. Dans sa conférence de presse du 14 janvier 1963, de Gaulle réagit de façon impitoyable au 'complot anglo-saxon'. Il déclare que l'entrée de la Grande-Bretagne changerait complètement le visage des Communautés et qu'en définitive, 'il apparaitraît une communauté atlantique colossale sous dépendance et direction américaines et qui aurait tôt fait d'absorber la communauté européenne' ${ }^{49}$.

Le même mois voit la signature du traité franco-allemand. La consultation politique que de Gaulle a initialement voulu organiser dans le cadre d'un directoire anglo-franco-américain, puis au niveau des Six, sera finalement organisée cinq ans plus tard avec l'Allemagne, mais ses ambitions seront beaucoup plus limitées. La cooptration franco-allemande sera le seul effet durable des discussions sur l'union politique européenne. Il s'agit d'un changement radical dans l'attitude de la France, qui en 1954 encore, avait saboté la ratification du traité établissant la Communauté européenne de Défense de peur de voir se développer une armée allemande trop forte. Au lieu de voir ce pays comme un rival, la France choisira de le mettre dans son giron pour pouvoir profiter de sa puissance et en même temps éviter qu'il ne s'écarte trop du rôle qu'elle veut lui voir jouer. Il faut attendre jusqu'en 1970, c'est-à-dire jusqu'à l'adoption du Rapport de Luxembourg sur la coopération politique, pour voir se réaliser le voeu d'étendre la coopération francoallemande aux autres partenaires de l'Europe. La voie intergouvernementale ne sera tependant pas abandonnée.

(49) R. BLOES, o.c., p. 417. 
Summary: The position of Belgium in the negotiations on the 'Fouchet Proposal'.

On 17 April 1962, General de Gaulle's proposal to establish a political union between the six EC Member States, better known as the 'Fouchet Proposal', was vetoed by Belgium and The Netherlands who made their further support dependent on British participation in the negotiations on a political union.

This article examines the position of Belgium, represented by its Minister for Foreign Affairs, Mr. P-H. Spaak and more particularly its evolution from a rather favourable position to a rejection of the project. The contradictory Belgian demand for a more supranational political union at the same time as British membership can be considered in the light of Belgium's wish to promote both the process of European integration and the principle of Atlantic solidarity, the basis of its security. The article describes how the final deathblow to the negotiations was not given by Belgium and The Netherlands but by the French veto of British accession to the EC and the signing of the Franco-German Cooperation Treaty of Elysee in January 1963. 